\title{
Konkurrierende Netzwerke
}

\author{
Da es keine Weltregierung gibt, die globale Gefährdungen abwenden könnte, \\ stellt sich die Frage, ob und wie transnationale "Governance ohne Govern- \\ ment" auf dem Umweltsektor möglich ist. Für bisherige Erfolge spielten \\ Politiknetzwerke und Wissenschaftler eine wichtige Rolle.
}

$\mathrm{D}$ Von Reiner Grundmann ie Fragestellung nach transnationalen Lösungspotentialen im Umweltbereich ist relativ neu (1). Die Problematik globaler Umweltgefährdungen kam erst zu Beginn der 70er Jahre durch die Berichte des Club of Rome und die Einrichtung des Umweltprogramms der Vereinten Nationen (UNEP) auf die Tagesordnung. Bis heute sind über 170 multilaterale, völkerrechtlich gültige Verträge auf dem Umweltsektor geschlossen worden. Zum Beispiel wird der Schutz der Ozonschicht durch nationale und internationale Regulierungen angestrebt, die bislang als erfolgreich gelten können. Die weltweit FCKW-Produktion ist von 1986 bis 1992 um über 50 Prozent zurückgegangen. Ein weiteres Beispiel ist das Klimaprotokoll von Kioto (vgl. Althammer in diesem Heft).

\section{$>$ Vier Hindernisse}

Es sind vor allem vier Punkte, die Erfolge auf dem Gebiet der internationalen Umweltpolitik unwahrscheinlich machen. Erstens ist die erfolgreiche Repräsentation diffuser Interessen zu nennen (2). Auf Basis des Theorems der rationalen Entscheidungswahl ist die Repräsentation von diffus verteilten Gemeinwohlinteressen gegenüber einer Industrie, auf die im Regulierungsfall Kosten zukommen, äußerst problematisch. Zweitens müssen im Unterschied zu Regulierungen im Rahmen des Nationalstaats, in dem der "Schatten der Hierarchie“ günstigere Bedingungen zur Regulierung abgibt, Regulierungen auf internationaler Ebene im ,Schatten der Anarchie" erfolgen. Zwar hat die neo-institutionalistische Richtung auf dem Gebiet der internationalen Beziehungen auf kooperationsfördernde Rahmenbedingungen aufmerksam gemacht (3). Doch sie geht entweder von Voraussetzungen aus, die nicht immer gegeben sind (meist wird das Modell des Gefangenendilemmas zugrundegelegt), oder sie setzt das zu Erklärende voraus, indem Kooperation als Ergebnis von internationalen Regimes gefaßt wird, wo es doch zuerst darum geht, das Entstehen eines internationalen Regimes zu erklären. Damit verbunden ist drittens die Problematik der öffentlichen Güter. Viele Umweltressourcen (wie die Erdatmosphäre, die Weltmeere, die biologische Vielfalt) sind Gemeinschaftsgüter und keine klassischen öffentlichen Güter. Dies ist keine Haarspalterei: der Unterschied ist wichtig, da er weitreichende Konsequenzen hat. Denn ein öffentliches Gut kann im Prinzip durch einen einzelnen Akteur bereitgestellt werden, ohne daß es durch das Trittbrettfahren anderer gefährdet wird. Im Gegensatz hierzu kann unilaterales Handeln eine Gemeischaftsressource nicht bereitstellen oder auch nur bewahren, wohl aber schädigen oder zerstören. Daraus folgt, daß alle potentiellen Schädiger in bindenden internationalen Abkommen zu bestimmten Maßnahmen verpflichtet werden müssen.

Noch unwahrscheinlicher wird erfolgreiche Kooperation auf dem Gebiet der internationalen Umweltpolitik, wenn man schließlich viertens bedenkt, daß politische Entscheidungen unter Unsicherheit erfolgen müssen. In solchen Situationen rückt Expertenwissen in eine zentrale Position (4). Es kann aber keine sichere Bewertung leisten, da typischerweise (mindestens) zwei gegensätzliche Experteneinschätzungen aufeinandertreffen (5). Wissenschaftliche Kontroversen sind mit politischen verflochten.

Welche Erklärungen gibt es angesichts dieser Hindernisse für die dennoch vorhandenen Erfolge?

\section{> Politiknetzwerke...}

Der Schlüssel zum Verständnis ist in der spezifischen institutionellen Form des Netzwerks zu suchen. Anders als in pluralistischen oder neokorporatistischen Ansätzen, in denen Politikergebnisse entweder durch das Wirken konstitutioneller Akteure oder mächtiger Interessengruppen erklärt und oft spieltheoretisch verfeinert werden, betrachtet der Politik- netzwerkansatz informelle Beziehungen zwischen Akteuren. Generell mobilisieren Netzwerke Akteure und Ressourcen, wobei die Spezifik der Umweltproblematik darin besteht, daß materielle Ressourcen vor allem zur Produktion symbolischer Ressourcen verwendet werden. Ideen fungieren dabei als Kondensationskerne, um die herum eine Anlagerung von Bündnispartnern erfolgt. Dadurch wird die Struktur eines Konflikts vorgezeichnet.

Konflikte über Fragen der Umwelt und Gesundheit entzünden sich im wesentlichen um Problemdefinitionen und Kausalrelationen. Dabei fungieren Informationen, wissenschaftliche Ergebnisse, Deutungen und Argumente als Primärressourcen. Dies hat einen Kampf um knappe (symbolische) Ressourcen zur Folge. Die (Un)Verfügbarkeit symbolischer Ressourcen befördert (oder behindert) die Wachstumschancen eines entsprechenden Politiknetzwerks. Öffentliche Glaubwürdigkeit ist bei Entscheidungen unter Unsicherheit eine der wertvollsten Ressourcen. Institutionelle Opportunitätsstrukturen (politische Verfassung, Politikstil, institutionelles Umfeld) können die Wachstumschancen ebenfalls befördern oder behindern. Erlangt ein Politiknetzwerk die Hegemonie, folgen in aller Wahrscheinlichkeit Regulierungen oder deren dauerhafte Abwehr. Strukturell gesehen geht es also um Akteurskonstellationen, in denen die Präferenzen der Akteure durch Macht, Interessen und Ideen bestimmt werden. Ideen spielen eine besondere Rolle: sie besteht darin, daß sie in Form von Informationen, Interpretationen und Normen Akteurspräferenzen mitbestimmen und ein ,Framing" von Situationen leisten (6). Bei Entscheidungen unter Unsicherheit gibt es mindestens zwei Akteursgruppen (,Lager“, Koalitionen), die auf Basis gegensätzlicher Normen entgegengesetzte Interpretationen der Situation vornehmen. Je besser einer von ihnen das Framing gelingt, desto wahrscheinlicher, daß ihre Position innerhalb der Politikarena und in der breiten Öffentlichkeit als die glaubwürdigere erscheint.

\section{-...und Unsicherheit}

In Politikfeldern, in denen Unsicherheitsreduktion zentral ist, bilden sich (idealtypisch vereinfacht) zwei Politiknetzwerke mit konträren Zielen heraus, die mit den Mitteln der Wissenschaft um gesellschaftspolitische Ziele kämpfen. Ein Politiknetzwerk befürwortet schnelle Regulierungen auch wenn wissenschaftliche Unsicher- 
heiten fortbestehen, das andere verlangt zuerst eine umfassende Klärung aller Fragen. Diese beiden Grundhaltungen beruhen auf prinzipiellen, normativen Orientierungen, die in der Kontroverse nicht zur Disposition stehen, dem Vorsorgeprinzip und dem Abwarteprinzip. Beide Seiten verfügen über Sprecher, die eine Verknüpfung von wissenschaftlichen Daten mit Politikzielen herstellen und sich für diese in der Öffentlichkeit einsetzen. Dabei versuchen sie, Ressourcen und kontingente Umweltereignisse als Mittel für ihre Ziele zu mobilisieren. Sobald die Repräsentation diffuser Interessen durch Sprecher erfolgt, müssen auch die organisierten Interessen durch Sprecher auftreten. Dadurch wird der strukturelle Ausgangsvorteil organisierter Interessen relativiert. Dies ist ein wesentlicher Hebel, um diffuse Interessen zu artikulieren und in den politischen Prozeß einzuschleusen. Die Hebelwirkung entsteht, da in der öffentlichen Debatte Argumente dem Zwang unterliegen, sich so darstellen zu müssen, daß sie allgemein akzeptabel sind. Die Industrie kann daher nicht nur auf der Verteidigung ihrer Partialinteressen bestehen.

\section{Gesellschaftspolitische Rolle von Wissenschaftlern}

Wenn Sprecher in solchen Kontroversen eine exponierte Stellung einnehmen und ihre Situationsdeutungen relevant sind, so rücken ihre Interpretationen und Rhetorik in den Mittelpunkt des Interesses. Quantität und Qualität der in Frage stehenden Expertenmeinung werden zum Gegenstand der Kontroverse: Wieviele Wissenschaftler vertreten eine bestimmte Position und wie glaubwürdig ist diese? Gelingt es, viele Wissenschaftler für eine Position zu mobilisieren, so kann dieser Mengeneffekt als Indikator für Konsens gedeutet werden, auch wenn keine wissenschaftlichen Beweise erbracht wurden (7). Da Expertenurteile immer vorgeben, auf wissenschaftlichen Daten und Theoremen zu basieren, spielt sich ein Teil des Streits darüber ab, was „gute" Wissenschaft ist (oder was überhaupt als Wissenschaft gelten kann).

Es sind engagierte Atmosphärenwissenschaftler, die Öffentlichkeit und Politik besonders erfolgreich alarmieren und beim Aufbau eines wissenschaftlich-politischen, transnationalen Netzwerks eine wesentliche Rolle spielen. Einige der bekannten Namen aus dem Bereich der Atmosphärenwissenschaften sind Bert Bolin, Paul Crutzen, Hartmut Graßl, Sherry Rowland und
Robert Watson. Die Mobilisierung korporativer Akteure, materieller und symbolischer Ressourcen sind die schlagenden „Argumente“, die die Politik von der Notwendigkeit des Handelns, nicht zuletzt im Kampf um Wählerstimmen, überzeugen kann. Nicht immer finden diese Appelle Gehör bei der Politik. Treten jedoch keine engagierten Wissenschaftler auf, so darf vermutet werden, daß die zugrundeliegenden Probleme kaum eine Chance haben, auf die politische Tagesordnung zu gelangen.

Wissenschaftlich-politische Kontroversen entwickeln sich in jedem der an einem internationalen Umweltabkommen beteiligten Länder in unterschiedlicher Weise. In jedem Land gibt es ein anderes Kräfteverhältnis zwischen Regulierungsbefürwortern und -gegnern. Erfolgreiche Kooperation setzt voraus, daß die Regulierungsbefuirworter in allen wichtigen Ländern die Oberhand gewinnen. Dies beginnt meist mit Akten der Selbstbindung, entweder unilateralen Regulierungen oder der Absichtserklärung. Die Chancen, solche Brückenköpfe auszubauen, werden im wesentlichen durch innenpolitische Faktoren in jedem Land bestimmt, aber auch durch das Wirken der transnationalen Politiknetzwerke, die eine gezielte Beeinflussung der öffentlichen Meinung in verschiedenen Ländern anstreben. Besonders effektiv ist ein Mobilisierungsmechanismus, bei dem Wortführer aus der Allianz der Regulierungsgegner neutralisiert werden oder dem Pro-Regulierungs-Lager zugefuihrt werden.

Der erfolgreiche Abschluß des Verhandlungsprozesses zum Schutz der Ozonschicht und der relative Erfolg der Klimaschutzmaßnahmen sind auf eine Kombination dieser beiden Faktoren zurïckzufïhren. Beim Zustandekommen des Montrealer Protokolls zum Schutz der Ozonschicht war es vor allem die veränderte Haltung der Europäischen Gemeinschaft, die sich schließlich dem amerikanischen Druck beugte und weitgehenden Maßnahmen zustimmte. Dabei hat vor allem der Positionswechsel der Bundesregierung eine Rolle gespielt. Beim Zustandekommen des Protokolls von Kioto war es vor allem das Entgegenkommen der USA, das allerdings unter Vorbehalt der Zustimmung des US-Kongresses steht. Im ersten Fall hatten die USA bereits unilaterale Maßnahmen zur Reduktion von FCKW ergriffen (Clean Air Act von 1977), im zweiten Fall hatte die EU ein anspruchsvolles Kohlendioxid-Reduktionsziel formuliert.
Die besonderen Eigenschaften des Politikfelds globale Umweltprobleme erfordern eine besondere Analyse. Die Problematik der Artikulation diffuser Interessen und politische Entscheidungen unter Unsicherheit lassen eigentlich vermuten, daß die Chancen für eine internationale Kooperation schlecht stehen. Engagierte Wissenschaftler, die sich aus ihrer Fachdisziplin herauswagen und eine öffentlich-politische Rolle spielen, können hier Abhilfe schaffen, vor allem, wenn sie hoch reputierten Forschungsinstitutionen angehören. Ihnen gelingt es zuweilen, diffuse Interessen an der Bewahrung der natülichen Umwelt und am Schutz menschlicher Gesundheit zu repräsentieren, wobei sie zweifelsohne von ihrer Rolle als autoritativer Stimme (der Wissenschaft) profitieren. Solche Stellungnahmen sind im politischen Prozeß auch dann einflußreich, wenn keine abschließenden Ergebnisse vorliegen. Die Mobilisierung durch Politiknetzwerke spielt dabei eine wesentliche Rolle.

\section{Anmerkungen}

(1) Der Begriff transnationale Beziehungen bezeichnet regelmäßige Interaktionen über Staatengrenzen hinweg, wobei mindestens ein nicht-staatlicher Akteur beteiligt sein muß. Im Unterschied dazu handelt es sich bei internationalen Beziehungen um solche zwischen Staaten.

Risse-Kappen, Thomas: Bringing Transnational Relations

Back in: Non-State-Actors, Domestic Structures, and International Institutions. New York 1995.

(2) Wilson, James Q.: The Politics of Regulation, New York 1980.

(3) Vor allem durch den Mechanismus der wiederholten Spiele, vgl. Axelrod, Robert: The Evolution of Cooperation. New York, 1984.

(4) Haas, Peter M.: Introduction: Epistemic Communities and International Policy Coordination. In: International Organization 46 (1992), S. 1-35.

(5) Jasanoff, Sheila: Science at the Bar: Law, Science, and Technology in America. Cambridge Ma, 1995.

(6) Rein, Martin, Donald Schön: Reframing Policy Discourse, in: Frank Fischer, John Forester (Hrsg.): The Argumentative Turn in Policy Analysis and Planning. Durham, London 1993, S. 145-166.

(7) Grundmann, Reiner: Politiknetzwerke und globale ökologische Probleme: Der Fall der Ozonschicht. In: Politische Vierteljahresschrift, 38 (1996), Nr. 2, S.247-273.

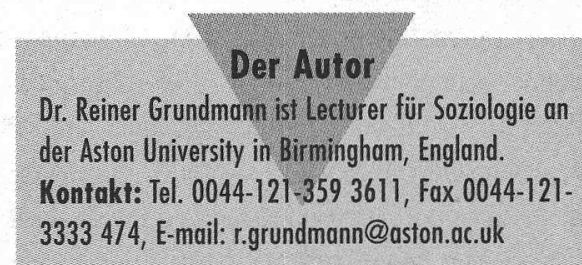


(c) 20I0 Authors; licensee IÖW and oekom verlag. This is an article distributed under the terms of the Creative Commons Attribution Non-Commercial No Derivates License (http://creativecommons.org/licenses/by-nc-nd/3.o/), which permits unrestricted use, distribution, and reproduction in any medium, provided the original work is properly cited. 